\title{
Anticholinergic activity disappears soon after the prescription of cholinesterase inhibitor
} \author{
Atsuko Inamoto ${ }^{2}$ \\ ${ }^{1}$ Department of Psychiatry, Showa University Northern Yokohama Hospital, Yokohama, Kanagawa, Japan \\ ${ }^{2}$ Department of Neuropsychiatry, St. Marianna University, School of Medicine, Kawasaki, Kanagawa, Japan \\ ${ }^{3}$ Tokyo Metropolitan Tobu Medical Center for Persons with Developmental/ Multiple Disabilities, Tokyo, Japan \\ ${ }^{4}$ Department of Pharmaceutical Therapeutics, Division of Clinical Pharmacy, School of Pharmacy, Showa University, Tokyo, Japan \\ ${ }^{5}$ Department of Psychiatry, Showa University East Hospital, Tokyo, Japan \\ ${ }^{6}$ Department of Anesthesiology, School of Medicine Juntendo University, Tokyo, Japan
}

Taishi Koganemaru ${ }^{1}$, Koji Hori ${ }^{1,2 *}$, Misa Hosoi ${ }^{1}$, Kimiko Konishi $^{3}$, Mitsugu Hachisu ${ }^{4}$, Hiroi Tomioka $^{1}$, Masayuki Tani $^{5}$, Yuka Kitajima ${ }^{6}$, and

\begin{abstract}
In this article we present the 80 years of woman patient who showed delusion, hallucination and excitement at night, those were thought to be related with the toxicity of anticholinergic activity (AA). These symptoms were resolved soon after by the prescription of Aricept ${ }^{\circledR}$ which is cholinomimetic agent. Because the disappearance of AA is relatively short time, we speculated that the disappearance of AA was related rather directory with upregulation of acetylcholine (ACh) not with antagonizing with AA by upregulation of ACh. From these speculation we are more convinced of the existence of our hypothesis, i.e., an 'endogenous anticholinergic cascade'.
\end{abstract}

Abbreviations: AA: anticholinergic activity; ACh : acetylcholine; AD: Alzheimer's disease; ChEI: cholinesterase inhibitor; LBD: Lewy body disease; SAA: serum anticholinergic activity

\section{Introduction}

We previously proposed the hypotheses of "endogenous appearance of Anticholinergic activity (AA) in Alzheimer's disease (AD) $[1,2]$ and in Lewy body disease (LBD) [3]. However, these hypotheses are merely "hypotheses". Therefore it is needed to prove these hypotheses. Recently, Yilmaz et al. [4] presented the case of a 19-year-old male who was brought to the emergency room for initial complaints of restlessness who was later diagnosed with intoxication of anticholinergic toxicity by herb medicine. Two milligrams of physostigmine were administered, which caused sudden recovery from the symptoms. From this case report, we are convinced of the existence of an 'endogenous anticholinergic cascade [1,2,5]' (Figure 1; courtesy of Hori et al. [5]). We also experienced the 80 years of woman patient whose clinical symptoms, those were thought to be related with AA, were resolved soon after by the prescription of Aricept $t^{\mathbb{B}}$ which is also cholinomimetic agent. In this article we present the case because we are more convinced of the existence of our hypothesis, i.e., an 'endogenous anticholinergic cascade [1,2,5]'. The patient and her proxy have provided written consent authorizing publication of this report.

\section{Case presentation}

Patients was 80 years old woman when she visited our hospital at first time (X years and Y months). AtX-20 years she felt digital numbness and anxiety after cerebral hemorrhage. Therefore, she was prescribed $2 \mathrm{mg}$ of etizolam. At X-3 years she also felt apathetic for cleaning her room. At X-2 years she was token mischief by neighborhood for some time. She has been delirious about her neighborhood. This delusion had been continued, therefore, at X years and Y months, she has been visited our hospital. We advised her to stop etizolam, but she could not stop because of her anxiety. She was also prescribed famotidine, however, true reason for this was not unknown. Even after visiting our hospital, she called for police because of mischief by neighborhood. Due to not ameliorating her symptoms, she was admitted to our hospital at $\mathrm{X}$ years and $\mathrm{Y}+1$ months. We aimed to provide feeling of safety by separating her form her neighborhood and to adjust her medicines. We stopped her etizolam suddenly and prescribed $25 \mathrm{mg}$ of quetiapine because etizolam poses anticholinergic property. However, her delusions had continued and she had exited, spoke loudly and was aggressive against the other patients and had been need to be restrained at night. In spite of this excitement at night, she had been relatively calm at daytime. She had seen her son who didn't visit the hospital. We started to prescribe $3 \mathrm{mg}$ of Aricept $\mathrm{t}^{\circledR}$ at $\mathrm{X}$ years, $\mathrm{Y}+1$ months and 14 days and increased Aricept ${ }^{\circledR} 5 \mathrm{mg}$ after 2 weeks. Next day of the prescription of Aricept $^{\mathbb{R}} 3 \mathrm{mg}$, she said "I mistook to think my neighborhood mischief me and my son to come to the hospital". Her symptoms of delusion, hallucination and excitement at night were disappeared since then. The score of mini-mental state examination (MMSE) [6,7] was 23 at study entry and the examination of cardiac 123-I-metaiodobenzylguanidine

Correspondence to: Koji Hori, M.D., Ph.D, St. Marianna University School of Medicine, Department of Neuropsychiatry, 2-16-1 Sugao Miyamaeku Kawasaki City, Kanagawa, Japan, Tel: +81-44-977-8111, E-mail: kojihori@marianna-u.ac.jp

Key words: acetylcholine (ACh), anticholinergic activity (AA), endogenous anticholinergic activity

Received: July 27, 2016; Accepted: August 05, 2016; Published: August 09, 2016 


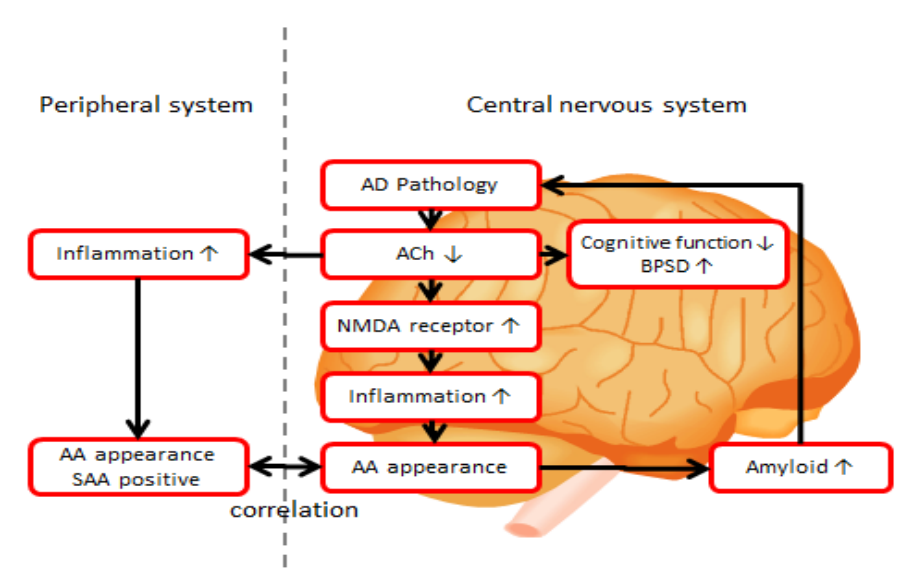

Figure 1. We speculate that the decrease in acetylcholine levels not only causes cognitive dysfunction and the BPSD but also allows for the inflammatory processes in the central nervous system and in the peripheral tissues. The latter changes bring about anticholinergic activity (AA) in both the central nervous system and peripheral tissues by cytokine activation. AA in turn promotes the buildup of amyloid, which further downregulates the cholinergic system. We call this vicious cycle an "endogenous AA cascade."

AA: anticholinergic activity, ACh: acetylcholine, AD: Alzheimer's disease, BPSD: behavioral and psychological symptoms of dementia, NMDA: N-methyl-D-aspartate, SAA: serum anticholinergic activity. This figure is from the article by Hori et al. [5].

(MIBG) scintigraphy showed that heart to mediastinum (H/M) ratio was 1.53 and washout rate was $35 \%$. Not $\mathrm{H} / \mathrm{M}$ ratio [8] but washout rate [9] was related with $\mathrm{LBD}$, from those we considered that the patient showed mild dysfunction of autonomic nervous system. From these results we diagnosed her LBD and prescribe her only $10 \mathrm{mg}$ of Aricept $^{\circledR}$ and quetiapine was stopped now. There was no reoccurrence of clinical symptoms since then.

\section{Discussion}

We considered that the clinical symptoms such as visual hallucination, delusion and excitement at night (diurnal rhythm disturbance) in this patient were related with anticholinergic burden. We previously reported that when comparing 26 serum anticholinergic activity (SAA) positive patient group with 50 SAA negative patient group delusion, hallucination, diurnal rhythm disturbance and global cognitive dysfunction were more severe, especially delusion and diurnal rhythm disturbance were independently related with SAA [10]. In fact, it is reported that anticholinergic burden is related memory dysfunction $[11,12]$, however, there was also reported that AA was related rather with psychotic symptoms not with cognitive dysfunction $[13,14]$. Mulsant et al. reported that AA was related with delusion [15]. Based on these reports including ours we considered the clinical symptoms in this patient were induced by AA.

Moreover, we considered that characteristics of our patient were similar with those of Yilmaz et al. in view of three points. As noted before Yilmaz et al. [4] presented the case of a 19-year-old male who was diagnosed with intoxication of anticholinergic toxicity. Two milligrams of physostigmine (cholinomimetic agent) were administered, which caused sudden recovery from the symptoms.

Firstly we speculate that ACh was not fully intact in our patient because the global cognitive function of our patient was etwas deteriorated, which was showed the fact that scores of MMSE in our patient was 23. Yilmaz et al. also commented that their patient's cholinergic system was not fully mature because his age was 19 . Second, $\mathrm{AA}$ was induced by other AA insert based on the base of downregulation of ACh. AA was reported that medication [16], physical illness [17] or mental stress [18] causes AA. However, we speculated that when the ACh system is intact and not depressed or overloaded, upregulation of $\mathrm{ACh}$ is possible even if another AA insert is present. Consequently, the inflammatory system is not upregulated, and AA does not appear [1]. Medicine with AA caused AA toxicity in patient of Yilmaz et al. and mental stress we speculated caused AA toxicity in our patient of course based on the not full intact ACh system. Thirdly Our patient was also suddenly ameliorated by the prescription of Aricept ${ }^{\mathbb{B}}$ because on the next day of first prescription of Aricept ${ }^{\mathbb{B}}$ clinical symptom related with AA in our patient disappeared, which is also similar with the patient in Yilmaz et al. Because the disappearance of AA is relatively short time in the patient of Yilmaz et al. and our patient, we speculated that the disappearance of AA was related rather directory with upregulation of ACh not with antagonizing with AA by upregulation of ACh. If the disappearance of AA had been related with antagonizing with AA by upregulated $\mathrm{ACh}$, repetitive and relative long term of the prescription of cholinesterase inhibitors were needed. From these speculations we are more convinced of the existence of our hypothesis, i.e., an 'endogenous anticholinergic cascade $[1,2,5]$ '.

Our study had main two limitations. Firstly we didn't measure SAA in this patient. Therefore the toxicity of AA was not definitely done in our patient. Secondary from the clinical features and MIBG findings that showed mild dysfunction of autonomic nervous system, we diagnosed her LBD. However, AD and stress response were not fully denied. Based on the diagnosis of LBD, we prescribed her Aricept ${ }^{\circledR}$. In Japan only Aricept ${ }^{\circledR}$ (original medicine of donepezil) is permitted for LBD. The generic medicines of donepezil are not permitted for LBD. Nevertheless, our patient is precious case that suggested 'endogenous anticholinergic cascade' as with the patient of Yilmaz et al.

\section{Conflict of interest}

Koji Hori received lecture fees from Eisai Co. Ltd., Pfizer Japan Inc., Novartis Pharma KK, Daiichi Sankyo Inc., Ono Pharmaceutical Co. Ltd., Janssen Pharmaceutical KK, Yoshitomi Yakuhin Co. Meiji Seika Pharma Co. Ltd., and Mitsubishi Tanabe Pharma Co. Mitsugu Hachisu received lecture fees from Meiji Seika Pharma Co. Ltd. and Mitsubishi Tanabe Pharma Co. However, the sponsors had no role in study design, data collection and analysis, decision to publish, or preparation of the manuscript.

\section{Disclosure statement}

Koji Hori received funding from by Eisai Co. Ltd., Daiichi Sankyo Inc. and Ono Pharmaceutical Co. Ltd. Mitsugu Hachisu received funding from Astellas Pharma Inc., Meiji Seika Pharma Co. Ltd., Dainippon Sumitomo Pharm Co. Ltd., Eli Lilly Japan KK, and Shionogi \& Co. Ltd.

\section{Author contributions}

Taishi Koganemaru was chief doctor for this patient. Koji Hori mainly coordinates the study regarding to anticholinergic activity. Misa Hosoi, Kimiko Konishi, Mitsugu Hachisu, Hiroi Tomioka, Masayuki Tani, Yuka Kitajima all members in Showa University Dementia Study Group and checked this manuscript. Hiroi Tomika advised and orgernized the clinical practice of this patient. Kimiko Konishi, Mitsugu Hachisu, Hiroi Tomioka, Masayuki Tani, and Yuka Kitajima wrote articles related with AA before. Atsuko Inamoto is chief professor in Showa University Northern Yokohama Hospital Department of Psychiatry also advised and orgernized the clinical practice of this patient and checked this manuscript. 


\section{References}

1. Hori K, Konishi K, Tani M, Tomioka H, Akita R, et al. (2014) Serum anticholinergic activity: a possible peripheral marker of the anticholinergic burden in the central nervous system in Alzheimer's disease. Dis Markers 2014: 459013. [Crossref]

2. Konishi K, Hori K, Tani M, Tomioka H, Kitajima Y, et al. (2015) Hypothesis of endogenous anticholinergic activity in Alzheimer's disease. Neurodegener Dis 15: 149-156.

3. Kitajima Y, Hori K, Konishi K, Tani M, Tomioka H, et al. (2015) A Review of the Role of Anticholinergic Activity in Lewy Body Disease and Delirium. Neurodegener Dis 15: 162-167. [Crossref]

4. Yilmaz MS, Isik B, Ongar M, Kavalci C, Solakoglu AG, et al. (2014) Delirium due to Datura stramoniun ingestion: a case report. Adv Res 2: 523-527.

5. Hori K, Konishi K, Akita R, Tani M, Tomioka H, et al. (2013) [Proposal of endogenous anticholinergic hypothesis in Alzheimer disease]. Nihon Shinkei Seishin Yakurigaku Zasshi 33: 117-126. [Crossref]

6. Folstein MF, Folstein SE, M Hugh RP (1975) "Mini-Mental State." A practical method for grading the cognitive state of patients for the clinician. J Psychiatry Res 12: 95-112. [Crossref]

7. Kato S, Shimogaki H, Onodera A, Ueda H, Oikawa K, et al. (1991) Development of the revised version of Hasegawa's Dementia Scale (HDS-R) (in Japanese). Jpn J Geriatric Psychiatry 2: 1339-1347.

8. Maruyama Y, Yamada T, Murakami K, Kumano R (2015) Comparison of the diagnostic performance of $\mathrm{H} / \mathrm{M}$ ratio between early and delayed phases for Lewy body disease. Nucl Med Commun 36: 477-480. [Crossref]

9. Lamotte G, Morello R, Lebasnier A, Agostini D, Defer GL (2015) Accuracy and cutoff values of delayed heart to mediastinum ratio with (123)I-metaiodobenzylguanidine cardiac scintigraphy for Lewy body disease diagnoses. BMC Neurol 15: 83. [Crossref]

10. Hori K, Konishi K, Watanabe K, Uchida H, Tsuboi T, et al. (2011) Influence of anticholinergic activity in serum on clinical symptoms of Alzheimer's disease. Neuropsychobiology 63: 147-153. [Crossref]

11. Sunderland T, Tariot PN, Cohen RM, Weingartner H, Mueller EA 3rd, et al. (1987) Anticholinergic sensitivity in patients with dementia of Alzheimer type and agematched control: a dose-response study. Arch Gen Psychiatry 44: 418-426. [Crossref]

12. Thienhaus OJ, Allen A, Bennett JA, Chopra YM, Zemlan FP (1990) Anticholinergic serum levels and cognitive performance. Eur Arch Psychiatry Clin Neurosci 240: 28 33. [Crossref]

13. Cummings JL1 (2000) Cholinesterase inhibitors: A new class of psychotropic compounds. Am J Psychiatry 157: 4-15. [Crossref]

14. Lemstra AW, Eikelenboom P, van Gool WA (2003) The cholinergic deficiency syndrome and its therapeutic implications. Gerontology 49: 55-60. [Crossref]

15. Mulsant BH, Pollock BG, Kirshner M, Shen C, Dogde H, et al. (2003) Serum anticholinergic activity in a community-based sample of older adults: relationship with cognitive performance. Arch Gen Psychiatry 60: 198-203. [Crossref]

16. Tune L, Carr S, Hoag E, Cooper T (1992) Anti-cholinergic effects of drugs commonly prescribed for the elderly: potential means for assessing risk of delirium. $\mathrm{Am} \mathrm{J}$ Psychiatry 149: 1393-1394. [Crossref]

17. Flacker JM, Lipsitz LA (1999) Serum anticholinergic activity changes with acute illness in elderly medical patients. J Gerontol A Biol Sci Med Sci 54: M12-16. [Crossref]

18. Plaschke K, Kopitz J, Mattern J, Martin E, Teschendorf P (2010) Increased cortisol levels and anticholinergic activity in cognitively unimpaired patients. JNeuropsychiatry Clin Neurosci 22: 433-441. [Crossref]

Copyright: (C2016 Koganemaru T. This is an open-access article distributed under the terms of the Creative Commons Attribution License, which permits unrestricted use, distribution, and reproduction in any medium, provided the original author and source are credited. 